\title{
THE APPLICATION OF BOOLEAN ALGEBRA IN MODELLING OF LEAKAGE CONDITION OF A CAR HYDRAULIC BRAKING SYSTEM
}

\author{
A. IDZIKOWSKI ${ }^{*}$ and S. SALAMON \\ Częstochowa University of Technology \\ Armii Krajowej str. 19 B, Częstochowa, POLAND \\ E-mail: adam.idzikowski@poczta.fm \\ salamon@zim.pcz.czest.pl
}

\begin{abstract}
A general characteristics of a car hydraulic braking system (CHBS) is presented in this publication. A graphical model of properties-component objects is developed for the above-mentioned system. Moreover, four mathematical models in terms of logic, the set theory and the Boolean algebra of Boolean functions are developed. The examination is ended with a general model of the CHBS for $n$ - Boolean variables and the construction and mathematical-technical interpretation of this model is presented.
\end{abstract}

Key words: braking system, hydraulic circuit, leakage, Boolean algebra, modelling.

\section{Introduction}

The braking system in one of the most important elements of a vehicle and its proper and effective functioning is the basic element of road traffic safety. A solution consisting in hydraulic transmission of force from a brake pedal to the mechanisms which brake individual wheels appeared in the automotive technique over 80 years ago.

Using one of the rules of the construction safety theory, i.e., the parametric excess, a dual-circuit braking system is applied. This system has no disadvantages which are characteristic of a single-circuit braking system. A basic disadvantage of the single-circuit system is a decrease or loss of braking force in the case of leakage or when the system is air-locked. In order to maintain a part of capabilities of the braking system in such a case, it is divided into two independent sections: one section operates the front axle wheels, and the other the rear axle wheels.

A design-constructional identification has been made for the capabilities of the car hydraulic braking system. This identification was limited to the main hydraulic circuit which is the most important part of this system in respect of reliability.

\section{Graphical model of "properties-component objects" for the structure of car hydraulic braking system}

A design-constructional identification has been made for the car hydraulic braking system. A simplified schematic-constructional diagram of this system is presented in Fig. 1 and it is limited to the main hydraulic circuit, which is the most important part of the braking system in respect of reliability. The presentation of this circuit was reduced to its most important components, i.e., the brake master cylinder and brake operating cylinders (Pihowicz, 2005).

A modern methodology of designing, documentation, analysis and systems management offers a lot of formal tools for searching for economical and equivalence-maintaining information. This methodology

\footnotetext{
* To whom correspondence should be addressed
} 
allows giving information the priorities or queuing sense, classifying and modifying it which is sometimes necessary and simultaneously optimal.

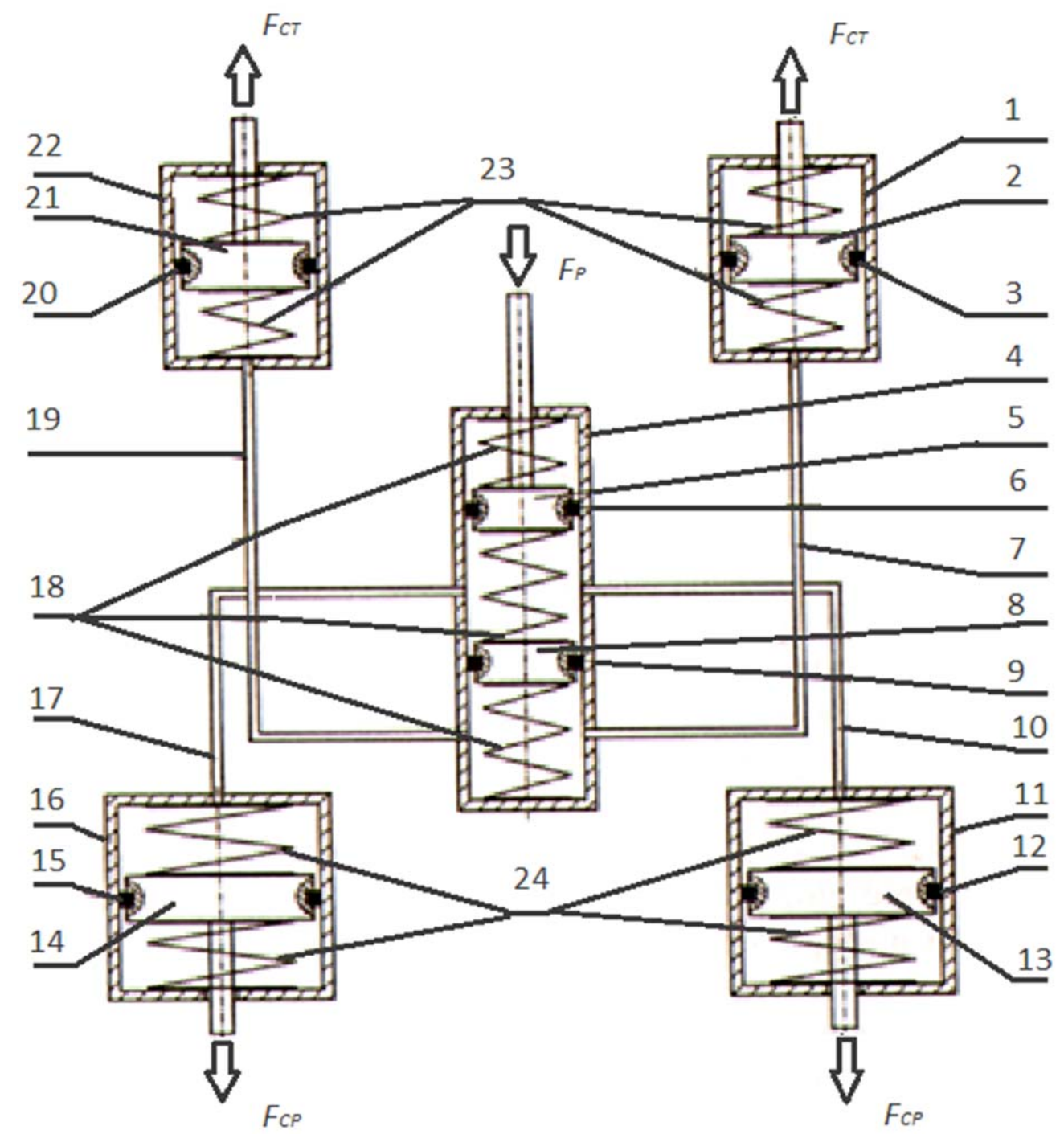

Fig.1. Schematic-constructional diagram of car hydraulic braking system - CHBS (Pihowicz, 2005) 1 - body of left rear brake operating cylinder, 2 - piston of left brake operating cylinder, 3 - seal of left rear brake operating cylinder piston, 4 - body of brake master cylinder, 5 - brake master cylinder rear piston, 6 - seal of brake master cylinder rear piston, 7 - left rear brake line, 8 - front piston of brake master cylinder, 9 - seal of brake master cylinder front piston, 10 - left front brake line, 11 - body of left front brake operating cylinder, 12 - seal of left front brake operating cylinder piston, 13 - piston of left front brake operating cylinder, 14 - piston of right front brake operating cylinder, 15 - seal of right front brake operating cylinder piston, 16 - body of right front brake operating cylinder, 17 - right front brake line, 18 - spacing springs of brake master cylinder, 19 - right rear brake line, 20 - seal of right rear brake operating cylinder, 21 - piston of right rear brake operating cylinder, 22 - body of right rear brake operating cylinder, 23 - return springs of rear brake operating cylinders, 24 - return springs of front brake operating cylinders, $\mathrm{Fp}$ - pressure on rear piston of brake master cylinder, $\mathrm{FcT}$ - pressure of rear brake operating cylinder piston on friction (brake) lining, $\mathrm{FcP}$ - pressure of front brake operating cylinder piston on friction (brake) lining. 
In particular, the diagnostic-functional strategies (Bieniak, 1999) and among them the techniques of information collection, have a significant meaning in the theory of effective organization and management (Mikołajczyk, 1994). One of them, i.e., the mapping technique (Martyniak, 1996), is a starting point for further examinations (Martyniak, 1996).

Taking into consideration the recommendations of this technique and being aware that a verbal record of the CHBS is often ambiguous and it can have only a supplemental character, both the schemes of components' construction and a scheme of the damages of the braking system hydraulic circuit have been used in this work. It should be emphasized, at this point, that they fulfil the essential criteria of a diagnostic method. They were subjected to a morphological analysis developed by F. Zwicky in 1948 (Martyniak, 1996).

The examinations were conducted in three stages:

1) determination of the problem,

2) analysis,

3) synthesis.

A graphical model of properties-component objects (Fig.2), being the vectors with two, three, five and 14 (as a target) components (Fig.3), has been developed on the basis of a design-constructional identification of the car hydraulic braking system.

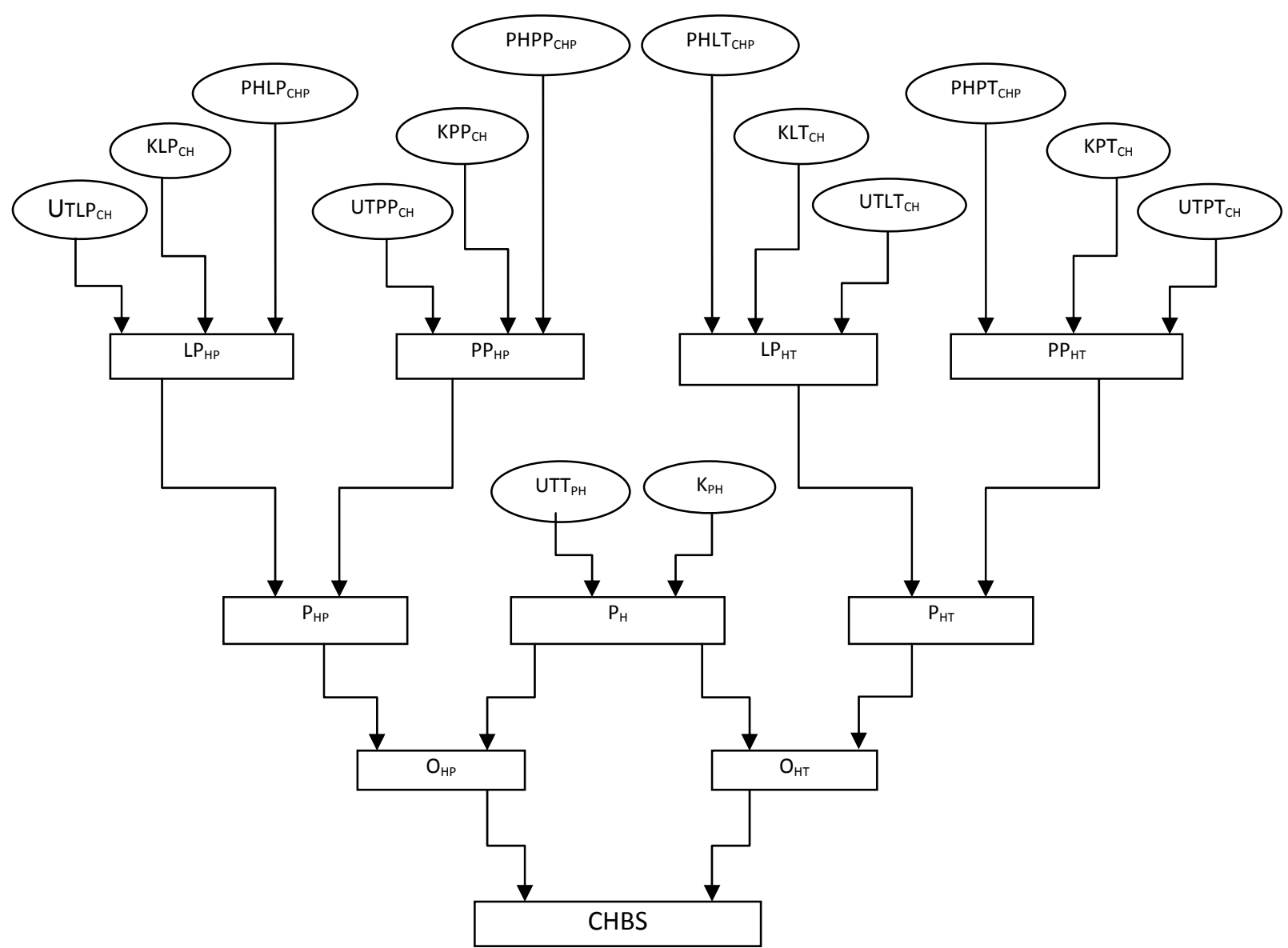

Fig.2. Graphical model of properties-component objects of the car hydraulic braking system (CHBS).

A graphical model of properties-component objects of the CHBS, including mathematical models on four levels of accuracy in terms of logic (Gubareni, 2001; Marek and Orzyszkiewicz, 2003; Rasiowa, 2004; Stupecki et al., 1994), the set theory (Kuratowski and Mostowski, 1978; Kuratowski, 2004; Marek and Orzyszkiewicz, 2003; Słupecki et al., 1994) and Boolean functions (Mostowski, 1964; Traczyk, 1970), is presented in Fig.3. 


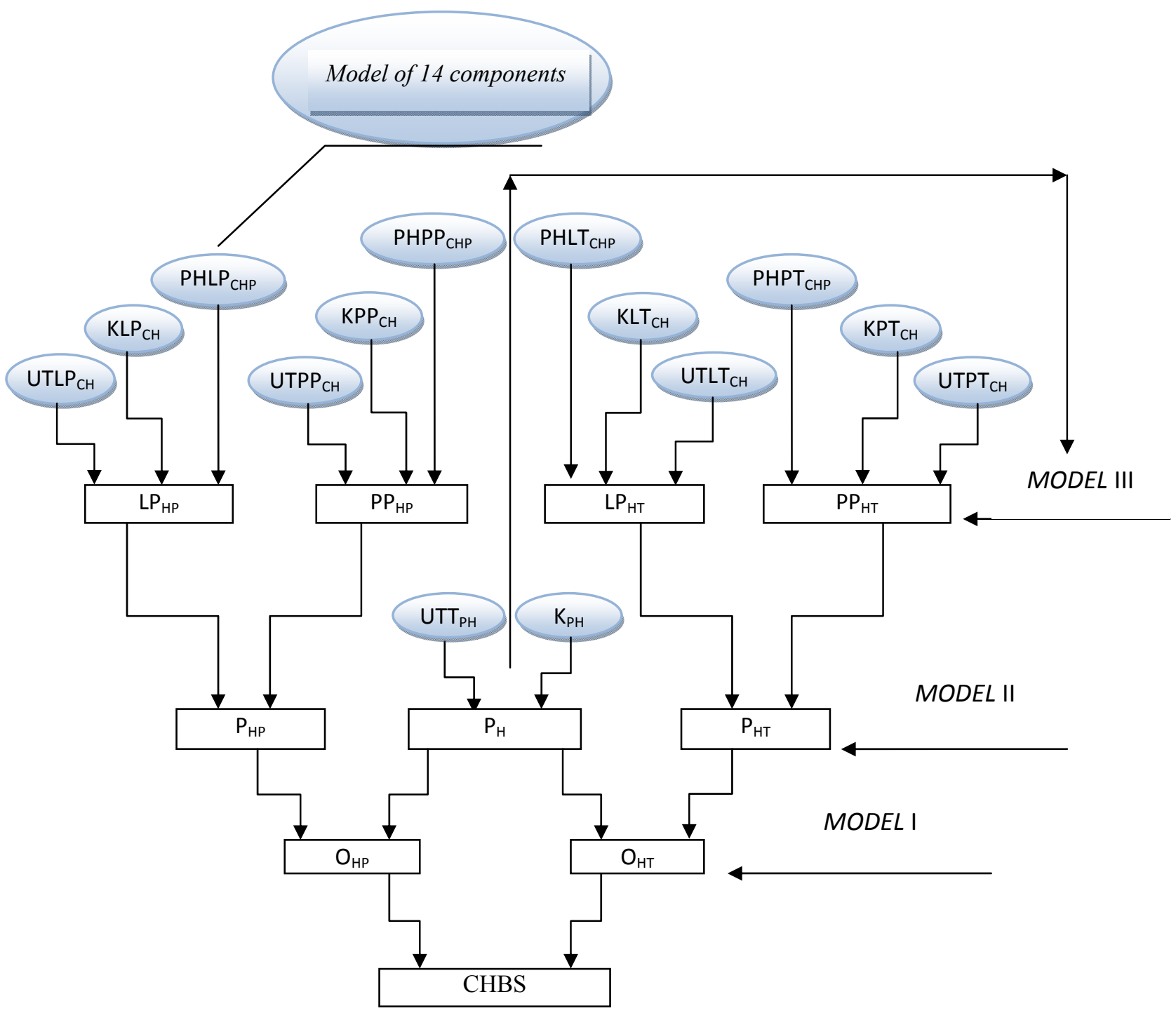

Fig.3. Graphical model of properties-component objects of the CHBS, including mathematical models on four sections of decomposition.

$O_{H P}=$ hydraulic object of front brakes, $O_{H T}=$ hydraulic object of rear brakes, $P_{H}=$ brake master cylinder, $P_{H P}=$ hydraulic sub-circuit (outside the brake master cylinder) of front brakes, $P_{H T}=$ hydraulic sub-circuit (outside the brake master cylinder) of rear brakes, $P P_{H P}=$ right hydraulic sub-circuit (outside the brake master cylinder) of front brakes, $L P_{H P}=$ left hydraulic sub-circuit (outside the brake master cylinder) of front brakes, $P P_{H T}=$ right hydraulic sub-circuit (outside the brake master cylinder) of rear brakes, $L P_{H T}=$ left hydraulic sub-circuit (outside the brake master cylinder) of rear brakes, $U T T_{P H}=$ seals of brake master cylinder rear piston, $K_{P H}=$ body of brake master cylinder, $U T P P_{C H}=$ seals of right front brake operating cylinder piston, $K P P_{C H}=$ body of right front brake operating cylinder, $P H P P_{C H P}=$ brake line connecting right front brake operating cylinder with brake master cylinder, $U T L P_{C H}=$ seals of left front brake operating cylinder piston, $K L P_{C H}=$ body of left front brake operating cylinder, $P H L P_{C H P}=$ brake line connecting left front brake operating cylinder with brake master cylinder, $U T P T_{C H}=$ seals of right rear brake operating cylinder piston, $K P T_{C H}=$ body of right rear brake operating cylinder, $P H P T_{C H P}=$ brake line connecting right rear cylinder with brake master cylinder, $U T L T_{C H}=$ seals of left rear brake operating cylinder piston, $K L T_{C H}=$ body of left rear brake operating cylinder, $P H L T_{C H P}=$ brake line connecting left rear brake operating cylinder with brake master cylinder. 


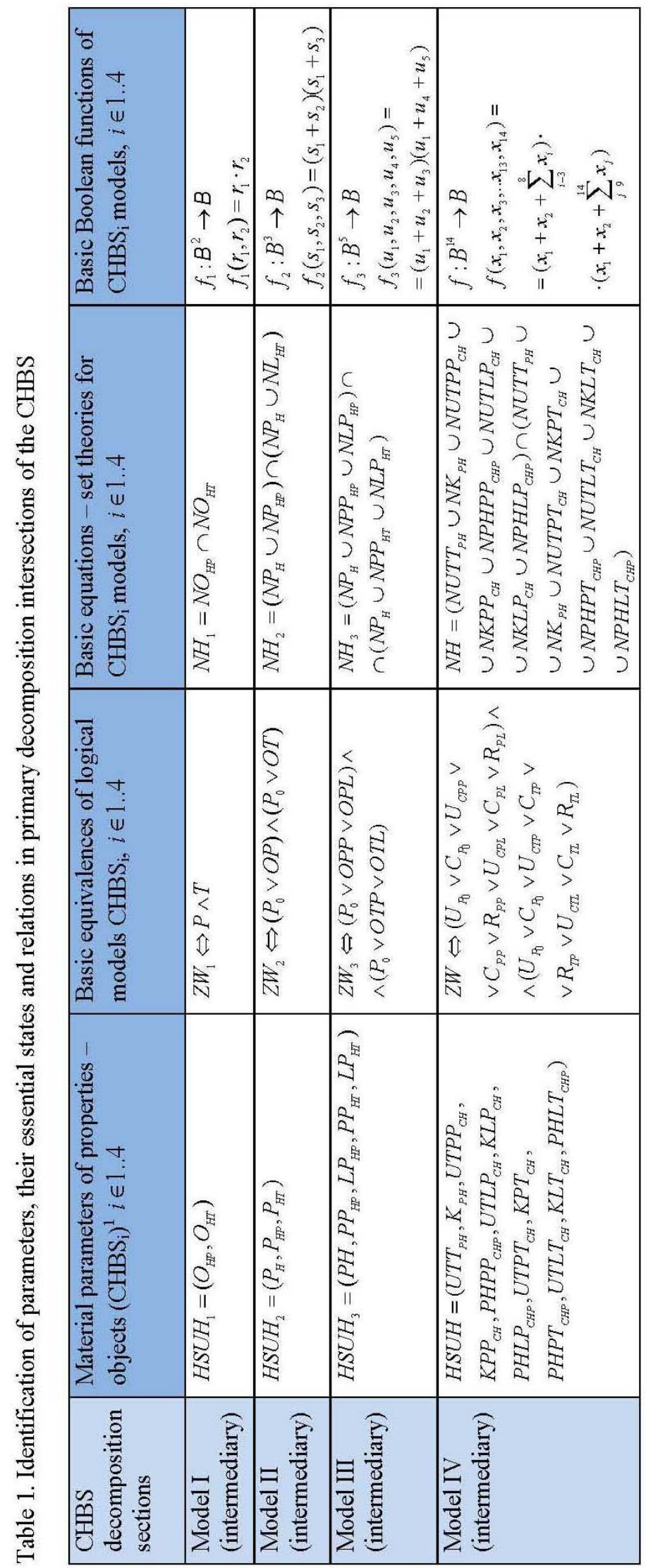

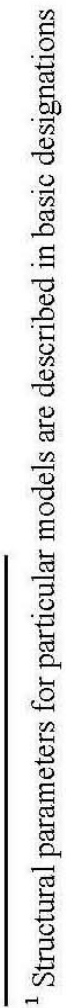


Taking into consideration the design-constructional identification of the structure of causal relationships between CHBS damages, the system of logical equivalences has been formulated on the set of logical sentences describing the events of CHBS damages. The list of sentences (and their designations) describing the intermediary and elementary events and regarding the damages of the system is included in the table of parameters identification and their essential states and relations in primary decomposition intersections of the CHBS (compare Tab.1).

The decomposition of the problem makes it possible to analyze it on various planes and from various points of view. The purpose of decomposition is to deepen the understanding of the problem and allow its logical defining. By analyzing the components distinguished in that manner and thus each level of problem complexity, one can examine and interpret the full structures of a problem better.

In the examinations and analyses conducted it was necessary to develop a general model for $n$ Boolean variables of the process of diagnosing the state of examined objects' unfitness (leakage) or fitness (leaktightness).

\section{General model of $\mathrm{CHBS}_{\mathrm{o}}$ in the Boolean algebra of Boolean functions}

The $\left(O_{p}\right)_{p=1}^{n}$ sequence of objects included in $\mathrm{CHBS}_{\mathrm{o}}$ and deciding about its leakage/leaktightness contains the following sub-sequences:

$\left(P_{H_{p}}\right)_{p=1}^{i}$ - elements of brake master cylinder,

$\left(O P P_{p}\right)_{p=i+1}^{i+j}$ - elements of hydraulic braking system of right side front axle,

$\left(O P L_{p}\right)_{p=i+j+1}^{i+2 j}$ - elements of hydraulic braking system of left side front axle,

$\left(O T P_{p}\right)_{p=i+2 j+1}^{i+3 j}$ - elements of hydraulic braking system of right side rear axle,

$\left(O T L_{p}\right)_{\substack{i+i+3 j+1 \\ p=i+3}}$ - elements of hydraulic braking system of left side rear axle

where:

$i=$ number of component elements influencing the leakage/leaktightness of brake master cylinder,

$j=$ number of component elements for each of OPP,OPL,OTP,OTL.

The sequence of Boolean variables $\left(x_{p}\right)_{p=1}^{n}, x_{p} \in\{0,1\}, p \in 1 . . n$

where

$$
x_{i}= \begin{cases}1, \text { object } O_{i} & \text { from }\left(O_{p}\right)_{p=1}^{n} \text { sequence is untight } \\ 0, \text { object } O_{i} & \text { from }\left(O_{p}\right)_{p=1}^{n} \text { sequence is tight }\end{cases}
$$

The Boolean function $f: B^{n} \rightarrow B$ determined by the Boolean formula for the leakage of the car hydraulic braking system $\left(\mathrm{CHBS}_{\mathrm{o}}\right)$ has the following form

$$
f(\boldsymbol{x})=\left(\sum_{p=1}^{i} x_{p}+\sum_{p=i+1}^{i+2 j} x_{p}\right)\left(\sum_{p=1}^{i} x_{p}+\sum_{r=i+2 j+1}^{i+4 j=n} x_{r}\right)
$$

for $\left(x_{s}\right)_{s=1}^{n} \in B^{n}$.

On the basis of the Boolean algebra properties, it follows 


$$
\begin{aligned}
& f(\boldsymbol{x})=[a(b+c)=a b+a c]=\sum_{p=1}^{i} x_{p} \sum_{p=1}^{i} x_{p}+\sum_{p=1}^{i} x_{p} \sum_{r=1+2 j+1}^{n} x_{r}+\sum_{p=1}^{i} x_{p} \sum_{q=i+1}^{i+2 j} x_{q}+ \\
& +\sum_{q=i+1}^{i+2 j} x_{q} \sum_{r=1+2 j+1}^{n} x_{r}=\left[\begin{array}{l}
a \cdot a=a \\
a+a b=a
\end{array}\right]=\sum_{p=1}^{i} x_{p}+\sum_{q=i+1}^{i+2 j} x_{q} \sum_{r=1+2 j+1}^{n} x_{r} .
\end{aligned}
$$

Taking into consideration both Boolean formulas for $f: B^{n} \rightarrow B$ and the determinations of Boolean variables assumed at the beginning, the following Boolean functions can be defined:

- the Boolean function of leakage in the brake master cylinder and hydraulic sub-circuit (outside the brake master cylinder) of front brakes

$$
f_{P P}(\boldsymbol{x})=\sum_{p=1}^{i} x_{p}+\sum_{q=i+1}^{i+2 j} x_{q}, \quad f_{p p}: B^{n} \rightarrow B
$$

- the Boolean function of leakage in the brake master cylinder and hydraulic sub-circuit (outside the brake master cylinder) of rear brakes

$$
f_{P T}(\boldsymbol{x})=\sum_{p=1}^{i} x_{p}+\sum_{r=i+2 j+1}^{i+4 j=N} x_{r}, \quad f_{P T}: B^{n} \rightarrow B
$$

- the Boolean function of leakage in the brake master cylinder

$$
f_{P H}(\boldsymbol{x})=\sum_{p=1}^{i} x_{p}, \quad f_{P H}: B^{n} \rightarrow B
$$

- the Boolean function of leakage in the hydraulic sub-circuit (outside the brake master cylinder) of front brakes

$$
f_{O P}(\boldsymbol{x})=\sum_{q=i+1}^{i+2 j} x_{q}, \quad f_{O P}: B^{n} \rightarrow B
$$

$f_{\text {OT }}(\boldsymbol{x})=f_{\text {OTP }}(\boldsymbol{x})+f_{\text {OTL }}(\boldsymbol{x})$, where:

- the Boolean function of leakage in the hydraulic sub-circuit (outside the brake master cylinder) of right side rear axle

$$
f_{\text {OTP }}(\boldsymbol{x})=\sum_{r=i+2 j+1}^{i+3 j} x_{r}: B^{n} \rightarrow B
$$

- the Boolean function of leakage in the hydraulic sub-circuit (outside the brake master cylinder) of left side rear axle

$$
f_{\text {OTL }}(\boldsymbol{x})=\sum_{r=i+3 j+1}^{i+4 j} x_{r}: B^{n} \rightarrow B
$$


- the Boolean function of leakage in the hydraulic sub-circuit (outside the brake master cylinder) of rear axle brakes

$$
f_{O T}(\boldsymbol{x})=\sum_{r=i+2 j+1}^{n} x_{r}, \quad f_{O T}: B^{n} \rightarrow B
$$

$f_{O P}(\boldsymbol{x})=f_{O P P}(\boldsymbol{x})+f_{O P L}(\boldsymbol{x})$, where:

- the Boolean function of leakage in the hydraulic sub-circuit (outside the brake master cylinder) of right side front axle brakes

$$
f_{O P P}(\boldsymbol{x})=\sum_{q=i+1}^{i+j} x_{q}: B^{n} \rightarrow B
$$

- the Boolean function of leakage in the hydraulic sub-circuit (outside the brake master cylinder) of left side front axle brakes

$$
f_{O P L}(\boldsymbol{x})=\sum_{q=i+j+1}^{i+2 j} x_{q}: B^{n} \rightarrow B
$$

Assuming the Boolean functions for $n$-variables in the form $B F_{n}=\left(F_{n},+, \cdot,-, 0,1\right)$, where $F_{n}=$ set of all Boolean functions of $n$ - Boolean variables, (operations on Boolean functions) $0=f(\boldsymbol{x})$ and $l=f(\boldsymbol{x})$ for $\boldsymbol{x} \in B^{n}$, the Boolean function $f \in F_{n}$ of $\mathrm{CHBS}_{\mathrm{o}}$ leakage is determined according to the following formula (3.1)

$$
f(\boldsymbol{x})=f_{P P}(\boldsymbol{x}) \cdot f_{P T}(\boldsymbol{x}) .
$$

In the generalized model of the CHBS the leakage can be computed as a product of the Boolean functions determined above or from the following formula (3.2)

$$
f(\boldsymbol{x})=f_{P H}(\boldsymbol{x})+f_{O P}(\boldsymbol{x}) \cdot f_{O T}(\boldsymbol{x}) .
$$

In the generalized Boolean model of the CHBS the leakage is the Boolean sum of Boolean functions of brake master cylinder leakages and the product of Boolean functions of leakages in the hydraulic circuits (outside the brake master cylinder) of front and rear brakes.

Substituting the component functions $f_{O P P}, f_{O P L}$ and $f_{O T P}, f_{O T L}$ to formula (3.13), we obtain

$$
\begin{aligned}
& f(\boldsymbol{x})=f_{P H}(\boldsymbol{x})+\left(f_{O P P}(\boldsymbol{x})+f_{O P L}(\boldsymbol{x})\right) \cdot\left(f_{O T P}(\boldsymbol{x})+f_{\text {OTL }}(\boldsymbol{x})\right)=[a(b+c)=a b+a c]= \\
& f_{P H}(\boldsymbol{x})+f_{O P P}(\boldsymbol{x}) \cdot f_{O T P}(\boldsymbol{x})+f_{O P P}(\boldsymbol{x}) \cdot f_{\text {OTL }}(\boldsymbol{x})+f_{\text {OPL }}(\boldsymbol{x}) \cdot f_{\text {OTP }}(\boldsymbol{x})+f_{O P L}(\boldsymbol{x}) \cdot f_{\text {OTL }}(\boldsymbol{x}) .
\end{aligned}
$$

Thus,

$$
\begin{aligned}
& f(\boldsymbol{x})=f_{P H}(\boldsymbol{x})+f_{O P P}(\boldsymbol{x}) \cdot f_{O T P}(\boldsymbol{x})+f_{O P P}(\boldsymbol{x}) \cdot f_{O T L}(\boldsymbol{x})+ \\
& +f_{O P L}(\boldsymbol{x}) \cdot f_{O T P}(\boldsymbol{x})+f_{O P L}(\boldsymbol{x}) \cdot f_{O T L}(\boldsymbol{x}) .
\end{aligned}
$$


In the technical interpretation, this equation in the Boolean algebra of Boolean functions and in morphological space $\mathrm{CHBS}_{\mathrm{o}}=(P H, O P P, O P L, O T P, O T L)$ confirms the known relationships in the structure of leakages which are determined by the leakages of all $\mathrm{CHBS}_{\mathrm{o}}$ components.

Continuing the above considerations for the function $f: B^{n} \rightarrow B$, the following results from formula (3.13) and De Morgan's laws are obtained:

$$
\begin{aligned}
& \bar{f}(\boldsymbol{x})=f_{S}(\boldsymbol{x})=\overline{f_{P H}(\boldsymbol{x})+f_{O P}(\boldsymbol{x}) \cdot f_{O T}(\boldsymbol{x})}=\left[\begin{array}{l}
\overline{a+b}=\overline{a b} \\
\overline{a b}=\bar{a}+\bar{b}
\end{array}\right]=\overline{f_{P H}}(\boldsymbol{x}) \cdot\left(\overline{f_{O P}}(\boldsymbol{x})+\overline{f_{O T}}(\boldsymbol{x})\right)= \\
& =[a(b+c)=a b+a c]=\overline{f_{P H}(\boldsymbol{x})} \cdot \overline{f_{O P}(\boldsymbol{x})}+\overline{f_{P H}(\boldsymbol{x})} \cdot \overline{f_{O T}(\boldsymbol{x})}= \\
& =\left[\begin{array}{l}
\overline{f_{P H}}=f_{S P H}: B^{n} \rightarrow B \text { - leaktightness function } P_{H}, \\
\overline{f_{O P}}=f_{S O P}: B^{n} \rightarrow B \text { - leaktightness function of front axle elements, } \\
\overline{f_{O T}}=f_{S O T}: B^{n} \rightarrow B \text { - leaktightness function of rear axle elements }
\end{array}\right]= \\
& =f_{S P H}(\boldsymbol{x}) \cdot f_{S O P}(\boldsymbol{x})+f_{S P H}(\boldsymbol{x}) \cdot f_{S O T}(\boldsymbol{x}) \text {. }
\end{aligned}
$$

The leaktightness function of the generalized CHBS model is the Boolean sum of the products of Boolean functions for brake master cylinder leakages and, respectively, the leaktightness of the hydraulic braking system of front and rear axles.

In the next morphological space of leakage/leaktightness of the general $\mathrm{CHBS}_{0}$ model, which includes (in addition to the brake master cylinder and hydraulic sub-circuits of brakes of both vehicles axles) also the significance of the leaktightness of brakes' hydraulic sub-circuit on left and right sides, the following functional equation can be formulated in the Boolean functions for $n$-variables

$$
\begin{aligned}
& \overline{f(\boldsymbol{x})}=\overline{f_{P H}(\boldsymbol{x})+\left(f_{O P P}(\boldsymbol{x})+f_{O P L}(\boldsymbol{x})\right) \cdot\left(f_{O T P}(\boldsymbol{x})+f_{O T L}(\boldsymbol{x})\right)}=\left[\begin{array}{l}
\overline{a+b}=\bar{a} \bar{b} \\
\overline{a b}=\bar{a}+\bar{b}
\end{array}\right]= \\
& =\overline{f_{P H}(x) \cdot\left(f_{O P P}(\boldsymbol{x})+f_{O P L}(\boldsymbol{x})\right)}+\overline{\left(f_{O T P}(\boldsymbol{x})+f_{O T L}(\boldsymbol{x})\right)}= \\
& \left.=\overline{f_{P H}(x)} \cdot \overline{\left(f_{O P P}(x)\right.} \cdot \overline{f_{O P L}(x)}+\overline{f_{\text {OTP }}(x)} \cdot \overline{f_{O T L}(x)}\right)= \\
& {[a(b+c)=a b+a c]=\overline{f_{P H}(\boldsymbol{x})} \cdot \overline{f_{O P P}(\boldsymbol{x})} \cdot f_{O P L}(\boldsymbol{x})+f_{P H}(\boldsymbol{x}) \cdot f_{O T P}(\boldsymbol{x}) \cdot f_{O T L}(\boldsymbol{x})=} \\
& \overline{f_{P H}}=f_{S P H}: B^{n} \rightarrow B \text { leaktightness function } P H \\
& \overline{f_{O P P}}=f_{S O P P}: B^{n} \rightarrow B \text { leaktightness function } O P P \\
& =\overline{f_{O P L}}=f_{S O P L}: B^{n} \rightarrow B \text { leaktightness function } O P L=f_{S P H}(\boldsymbol{x}) \cdot f_{S O P P}(\boldsymbol{x}) \cdot f_{S O P L}(\boldsymbol{x})+ \\
& \overline{f_{\text {OTP }}}=f_{\text {SOTP }}: B^{n} \rightarrow B \text { leaktightness function OTP } \\
& \overline{f_{\text {OTL }}}=f_{\text {SOTL }}: B^{n} \rightarrow B \text { leaktightness function OTL } \\
& +f_{S P H}(x) \cdot f_{\text {SOTP }}(x) \cdot f_{\text {SOTL }}(x) \text {. }
\end{aligned}
$$

Thus, 


$$
f_{S}(\boldsymbol{x})=f_{S P H}(\boldsymbol{x}) \cdot f_{S O P P}(\boldsymbol{x}) \cdot f_{S O P L}(\boldsymbol{x})+f_{S P H}(\boldsymbol{x}) \cdot f_{S O T P}(\boldsymbol{x}) \cdot f_{\text {SOTL }}(\boldsymbol{x})
$$

In the technical interpretation of the next morphological space, the leaktightness in $\mathrm{CHBS}_{\mathrm{o}}=(P H$, $O P P, O P L, O T P, O T L$ ) model occurs if and only if when $P H, O P P, O P L$ are tight simultaneously (brake master cylinder and hydraulic sub-circuit of both sides of the front vehicle axle) or when PH, OTP, OTL are tight simultaneously (brake master cylinder and hydraulic sub-circuit of both sides of the vehicle rear axle).

Based on the basic properties of the Boolean algebra in formula (3.2), we obtain

$$
\begin{aligned}
& f(\boldsymbol{x})=[a(b+c)=a b+a c]=\sum_{p=1}^{i} x_{p} \cdot \sum_{p=1}^{i} x_{p}+\sum_{p=1}^{i} x_{p} \sum_{r=1+2 j+1}^{n} x_{r}+\sum_{q=i+1}^{i+2 j} x_{q} \sum_{p=1}^{i} x_{p}+ \\
& +\sum_{q=i+1}^{i+2 j} x_{q} \sum_{r=1+2 j+1}^{n} x_{r}=\left[\begin{array}{l}
a \cdot a=a \\
a(b+c)=a b+a c
\end{array}\right]=\sum_{p=1}^{i} x_{p}+\sum_{p=1}^{i} \sum_{r=1+2 j+1}^{n} x_{p} x_{r}+ \\
& +\sum_{p=1}^{i} \sum_{q=i+1}^{i+2 j} x_{p} x_{q}+\sum_{q=i+1}^{i} \sum_{r=i+2 j+1}^{n} x_{q} x_{r}=\left[\begin{array}{l}
a+a b=a \\
a+a=a
\end{array}\right]=\sum_{p=1}^{i} x_{p}+\sum_{q=i+1}^{i=2 j} \sum_{r=i+2 j+1}^{n} x_{q} x_{r} .
\end{aligned}
$$

Formula (3.17) is the generalization of the Boolean formulas determining the leakage functions in all $\mathrm{CHBS}_{\mathrm{i}} / \mathrm{CHBS}, i \in 1 . .3$ Boolean models (for which it was proved in this work, by using the Quine McCluskey algorithm (Partyka, 1999), that they are MANF/IANF/SANF of these functions) onto $\mathrm{CHBS}_{\mathrm{o}}$.

\section{Summary}

The analysis of the hydraulic car brake system structure allowed determination of the decisive variables and relations between them in formal languages of logic, the set theory and the Boolean algebra of Boolean functions.

The level of accuracy of decisive variables has a cascade character, adequate to currently analyzed CHBS subsystem and its purpose is, inter alia, to identify the priority variables and formal relations among them. On the basis of structure analysis, the basic components of morphological space for three indirect mathematical models of the structure of leakage/leaktightness diagnosing process have been developed in formal languages of logic, the set theory and the Boolean algebra.

The Boolean functions $f, f_{S}: B^{14} \rightarrow B$ introduce a structure describing a natural initial diagnostics of the CHBS in respect of leakage/leaktightness of the brake master cylinder, the hydraulic sub-circuits (outside the brake master cylinder) of front and rear axle brakes and their both sides: left and right. Thus, they allow analyzing the issues of leakage/leaktightness in various intersections of accuracy determined during the examinations of initial models of the $\mathrm{CHBS}_{i}, i \in 1 . .3$ by the decomposition of the CHBS with the morphological method. The examinations were complemented by the development of a general model for $n$ - Boolean variables for the process of diagnosing leakage/leaktightness of the objects.

\section{Acronyms}

MANF - minimal alternative normal form, IANF - irreducible alternative normal form, SANF - shortened alternative normal form, CHBS - car hydraulic braking system. 


\section{References}

Bieniak H. (1999): Efficient Management Methods. - Ag. Ed. Placeta, Warsaw.

Gubareni N. (2001): Logic for Students. - Częstochowa: University of Częstochowa.

Kuratowski K. and Mostowski A. (1978): Set Theory. - Warsaw: PWN.

Kuratowski K. (2004): Introduction to Set Theory and Topology. - Warsaw: PWN.

Marek W. and Orzyszkiewicz J. (2003): Elements of Logic and Set Theory in Tasks. - Warsaw: PWN.

Martyniak Z. (1996): Organization and Management. - 60 Issues of Theory and Practice, Roman Publishing House, Kluczbork.

Mikolajczyk Z. (1994): Organizational Techniques to Solve Management Problems. - Warsaw: PWN.

Mostowski A.W. (1964): Boolean Algebras and their Applications. - Warsaw: PWN.

Partyka M.A. (1999): Quine McCluskey Algorithm to Minimize Individual Partial Multivalued Logic Functions, Studies and Monographs. - z 109, Publishing House, Opole.

Pihowicz W. (2005): Selected Aspects of Technical Safety Engineering - a Procedure for the Detection of Hazardous Areas in the Critical Components of Technical Objects. - Publishing House of Technical University of Wroclaw, Wroclaw.

Rasiowa H. (2004): Introduction to Contemporary Mathematics. - Warsaw: PWN.

Słupecki J., Hałkowska K. and Pirog Rzepecka K. (1994): Logic and Set Theory. - Warsaw: PWN.

Traczyk T. (1970): Introduction to the Theory of Boolean algebra. - Warsaw: PWN.

Received: September 23, 2012

Revised: February 8, 2013 